\title{
Applications and Solving Techniques of Propagated Wave in Waveguides Filled with Inhomogeneous Dielectric Materials
}

\author{
Zion Menachem
}

Additional information is available at the end of the chapter

http://dx.doi.org/10.5772/intechopen.76793

\begin{abstract}
This chapter presents techniques to solve problems of propagation along the straight rectangular and circular waveguides with inhomogeneous dielectric materials in the cross section. These techniques are very important to improve the methods that are based on Laplace and Fourier transforms and their inverse transforms also for the discontinuous rectangular and circular profiles in the cross section (and not only for the continuous profiles). The main objective of this chapter is to develop the techniques that enable us to solve problems with inhomogeneous dielectric materials in the cross section of the straight rectangular and circular waveguides. The second objective is to understand the influence of the inhomogeneous dielectric materials on the output fields. The method in this chapter is based on the Laplace and Fourier transforms and their inverse transforms. The proposed techniques together with the methods that are based on Laplace and Fourier transforms and their inverse transforms are important to improve the methods also for the discontinuous rectangular and circular profiles in the cross section. The applications are useful for straight waveguides in the microwave and the millimeter-wave regimes, for the straight hollow waveguide and for infrared field, also in the cases of inhomogeneous dielectric materials in the cross section.
\end{abstract}

Keywords: wave propagation, inhomogeneous dielectric materials, rectangular and circular waveguides, dielectric profiles

\section{Introduction}

The methods of straight waveguides have been proposed in the literature. Review of numerical and approximate methods for the modal analysis of general optical dielectric waveguides with 
emphasis on recent developments has been published [1]. Examples of important methods have been proposed such as finite-difference method, the integral-equation method, and methods based on series expansion. Full-vectorial matched interface and boundary method for modal analysis of dielectric waveguides has been proposed [2]. The method distinguishes itself with other existing interface methods by avoiding the use of the Taylor series expansion and by introducing the concept of the iterative use of low-order jump conditions.

A review of the hollow waveguide and the applications has been presented [3, 4]. A review of hollow waveguides, infrared transmitting, and fibers has been presented [5]. Hollow waveguides with metallic and dielectric layers have been proposed to reduce the transmission losses. A hollow waveguide can be made from any flexible or rigid tube, such as glass, plastic or glass, and the inner hollow surface is covered by a metallic layer and a dielectric overlayer. The structure of the layer enables to transmit both the TE and TM polarization with low attenuation [6, 7].

Selective suppression of electromagnetic modes in rectangular waveguides has been presented [8] by using distributed wall losses. Analytical model for the corrugated rectangular waveguide has been extended to compute the dispersion and interaction impedance [9].

A Fourier operator method has been used to derive for the first time an exact closed-form eigenvalue equation for the scalar mode propagation constants of a buried rectangular dielectric waveguide [10]. Wave propagation in an inhomogeneous transversely magnetized rectangular waveguide has been studied with the aid of a modified Sturm-Liouville differential equation [11]. A fundamental and accurate technique to compute the propagation constant of waves in a lossy rectangular waveguide has been proposed [12]. This method is based on matching the electric and magnetic fields at the boundary and allowing the wavenumbers to take complex values.

A method that relates to the propagation constant for the bound modes in the dielectric rectangular waveguides has been proposed [13]. An analysis of rectangular folded-waveguide slow-wave structure has been developed using conformal mapping by using Schwarz Christoffel transformation [14]. A simple closed form expression to compute the time-domain reflection coefficient for a transient $T E_{10}$ mode wave incident on a dielectric step discontinuity in a rectangular waveguide has been presented [15]. In this paper, an exponential series approximation was provided for efficient computation of the reflected and transmitted field waveforms.

The electromagnetic fields in rectangular conducting waveguides filled with uniaxial anisotropic media have been characterized [16]. In this paper, the electric type dyadic Green's function due to an electric source was derived by using eigenfunctions expansion and the Ohm-Rayleigh method. An improved generalized admittance matrix technique based on mode matching method has been proposed [17]. The generalized scattering matrix of waveguide structure and its discontinuity problems is obtained with relationship equations and reflection coefficients.

A full-vectorial boundary integral equation method for computing guided modes of optical waveguides has been proposed [18]. Method for the propagation constants of fiber waveguides 
of arbitrary cross section shape has been proposed [19]. In this paper, the proposed techniques used to solve problems of scattering by irregularly shaped dielectric bodies, and in the static limit, for solving the problem of an irregular dielectric or permeable body in an external field.

The rectangular dielectric waveguide technique for the determination of complex permittivity of a wide class of dielectric materials of various thicknesses and cross sections has been described [20]. In this paper, the technique has been presented to determine the dielectric constant of materials. The fields and propagation constants of dielectric waveguides have been determined within the scalar regime [21] by using two-dimensional Fourier series expansions. Propagation of modes in some rectangular waveguides using the finite-difference time-domain method has been proposed [22]. Analysis of rectangular waveguide using finite element method has been proposed [23].

Wave propagation and dielectric permittivity reconstruction in the case of a rectangular waveguide have been studied [24]. According to this paper, we study the electromagnetic wave propagation in a rectangular waveguide filled with an inhomogeneous dielectric material in the longitudinal direction. Light propagation in a cylindrical waveguide with a complex, metallic and dielectric function has been proposed [25]. Advancement of algebraic function approximation in eigenvalue problems of lossless metallic waveguides to infinite dimensions has been investigated [26]. The method of algebraic function approximation in eigenvalue problems of lossless metallic waveguides such as a closed uniform cylindrical waveguides has been proposed [27]. Analysis of longitudinally inhomogeneous waveguides using Taylor's series expansion has been proposed [28]. Analysis of longitudinally inhomogeneous waveguides using the Fourier series expansion has been proposed [29]. The method of external excitation for analysis of arbitrarily-shaped hollow conducting waveguides has been proposed [30].

A circular metallic hollow waveguide with inner dielectric multilayers has been designed by Miyagi and Kawakami [31] with the emphasis on low-loss transmission of the $H E_{11}$ mode for the infrared. According to this paper, the transmission losses of the dielectric-coated metal waveguides are drastically reduced when a multiple dielectric layer is formed instead of a single dielectric layer. The simplest and most efficient multilayer structure is a three-dielectriclayer stack deposited on a metal layer.

A transfer matrix function for the analysis of electromagnetic wave propagation along the straight dielectric waveguide with arbitrary profiles has been proposed [32]. According to this paper, the method is based on the Laplace and Fourier transforms, and the inverse Laplace and Fourier transforms. A rigorous approach for the propagation of electromagnetic fields along a straight hollow waveguide with a circular cross section has been proposed [33]. The cross section is made of a metallic layer, and only one dielectric layer upon it. The separation of variables is obtained by using the orthogonal-relations. The longitudinal components of the fields are developed into the Fourier-Bessel series. The transverse components of the fields are expressed as functions of the longitudinal components in the Laplace plane and are obtained by using the inverse Laplace transform by the residue method.

In order to solve more complex problems of coatings in the cross section of the dielectric waveguides, such as rectangular and circular profiles, then it is important to develop in each 
modal an improved technique to calculate the dielectric profile, the elements of the matrix and its derivatives of the dielectric profile.

The main objective of this chapter is to develop the techniques that enable us to solve problems with inhomogeneous dielectric materials in the cross section of the straight rectangular and circular waveguides. The second objective is to understand the influence of the inhomogeneous dielectric materials on the output fields. Thus, we need to develop the technique and a particular application to calculate the profiles in the cross section. Namely, we need to calculate the dielectric profile, the elements of the matrix and its derivatives of the dielectric profile in the cases of the straight rectangular and circular waveguides. The proposed techniques are important to improve the methods that are based on Laplace and Fourier transforms and their inverse Laplace and Fourier transforms also for the discontinuous rectangular and circular profiles in the cross section (and not only for the continuous profiles).

\section{Formulation of the problem}

In this chapter, we present techniques for solving discontinuous problems of dielectric materials in the cross section of the straight waveguide for applications in the microwave and millimeter-wave regimes and in the cases of infrared regime. The proposed techniques are very effective in relation to the conventional methods because they allow the development of expressions in the cross section only according to the specific discontinuous problem. In this way, the mode model method becomes an improved method to solve discontinuous problems in the cross section.

Three examples of inhomogeneous dielectric materials in the cross section of the straight waveguides are shown in Figure 1(a)-(c). Figure 1(a) shows an example of rectangular profile in the cross section of the straight rectangular waveguide. Figure 1(b) shows an example of

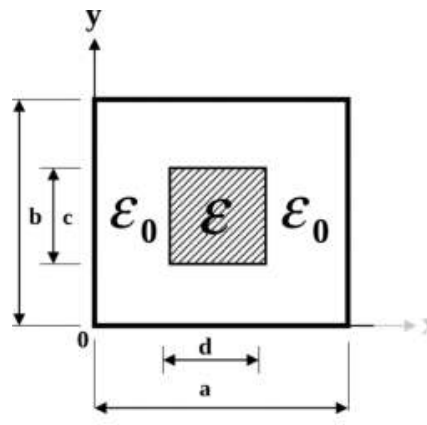

(a)

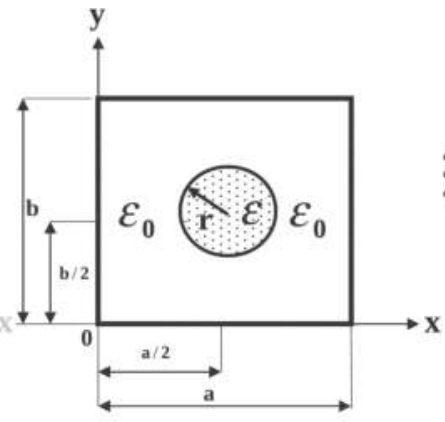

(b)

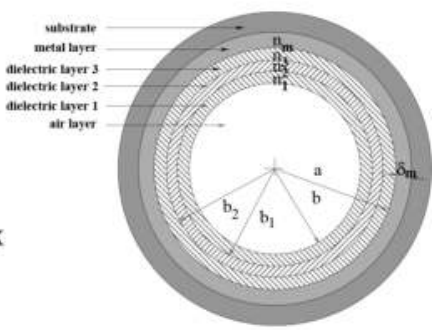

(c)

Figure 1. Three examples of inhomogeneous dielectric materials in the cross section of the straight waveguides. (a) Rectangular profile in the cross section of the straight rectangular waveguide; (b) Circular profile in the cross section of the straight rectangular waveguide; (c) Three dielectric layers and a metallic layer in the cross section of the hollow waveguide. 
circular profile in the cross section of the straight rectangular waveguide. Figure 1(c) shows an example for three dielectric layers and a metallic layer in the cross section of the straight hollow waveguide.

In order to solve these inhomogeneous dielectric materials, we need to calculate the dielectric profile, the elements of the matrix and its derivatives of the dielectric profile. In the next section, we explain the techniques to solve problems with inhomogeneous dielectric materials in the cross section of the straight rectangular waveguide (Figure 1(a) and 1(b)) and also in the cross section of the straight circular waveguide (Figure 1(c)).

\section{The technique to solve inhomogeneous dielectric profiles}

The particular application is based on the $\omega_{\varepsilon}$ function [34]. The $\omega_{\varepsilon}$ function is used in order to solve discontinuous problems of the rectangular profile, and circular profile in the cross section of the straight waveguide. The $\omega_{\varepsilon}$ function is defined as $\omega_{\varepsilon}(r)=C_{\varepsilon} \exp \left[-\varepsilon^{2} /\left(\varepsilon^{2}-|r|^{2}\right)\right]$ for $|r|>\varepsilon$, where $C_{\varepsilon}$ is a constant, and $\int \omega_{\varepsilon}(r) d r=1$. In the limit $\varepsilon \rightarrow 0$, the $\omega_{\varepsilon}$ function is shown in Figure 2 . The technique that based on $\omega_{\varepsilon}$ function is very effective to solve complex problems, in relation to the conventional methods, especially when we have a large numbers of dielectric layers and a metal layer, as shown in Figure 1(c). We will demonstrate how to use with the proposed technique for all the cases that are shown in the examples in Figure 1(a)-(c).

\subsection{The technique based on $\omega_{\varepsilon}$ function for the rectangular profile in the cross section of the straight rectangular waveguide}

The elements of the matrix $\mathrm{g}(\mathrm{n}, \mathrm{m})$ are calculated for an arbitrary profile in the cross section of the straight waveguide according to Figure 3(a) and 3(b). Figure 3(a) shows the arbitrary profile in the cross section of the straight waveguide. Figure $3(\mathbf{b})$ shows the rectangular profile in the cross section of the straight waveguide, according to Figure 1(a).

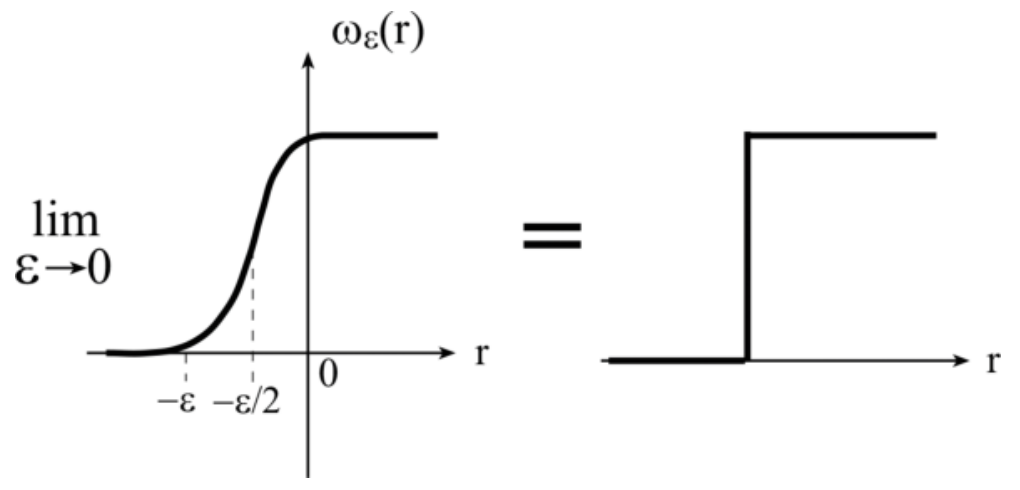

Figure 2. The technique based on $\omega_{\varepsilon}$ function in the limit $\varepsilon \rightarrow 0$ to solve discontinuous problems. 
The dielectric profile $g(x, y)$ is given according to $\varepsilon(x, y)=\varepsilon_{0}(1+g(x, y))$. According to Figure 3(a) and $3(\mathbf{b})$ and for $g(x, y)=g_{0}$, we obtain

$$
\begin{aligned}
g(n, m)= & \frac{g_{0}}{4 a b} \int_{-a}^{a} d x \int_{-b}^{b} \exp \left(-j\left(k_{x} x+k_{y} y\right)\right) d y \\
= & \frac{g_{0}}{4 a b}\left\{\int_{x_{11}}^{x_{12}} d x \int_{y_{11}}^{y_{12}} \exp \left(-j\left(k_{x} x+k_{y} y\right)\right) d y+\int_{-x_{12}}^{-x_{11}} d x \int_{y_{11}}^{y_{12}} \exp \left(-j\left(k_{x} x+k_{y} y\right)\right) d y\right. \\
& \left.+\int_{-x_{12}}^{-x_{11}} d x \int_{-y_{12}}^{-y_{11}} \exp \left(-j\left(k_{x} x+k_{y} y\right)\right) d y+\int_{x_{11}}^{x_{12}} d x \int_{-y_{12}}^{-y_{11}} \exp \left(-j\left(k_{x} x+k_{y} y\right)\right) d y\right\} .
\end{aligned}
$$

If $y_{11}$ and $y_{12}$ are not functions of $x$, then the dielectric profile is given by

$$
g(n, m)=\frac{g_{0}}{a b} \int_{x_{11}}^{x_{12}} \cos \left(k_{x} x\right) d x \int_{y_{11}}^{y_{12}} \cos \left(k_{y} y\right) d y .
$$

The derivative of the dielectric profile in the case of $y_{11}$ and $y_{12}$ are functions of $x$, is given by

$$
g_{x}(n, m)=\frac{2}{a m \pi} \int_{x_{11}}^{x_{12}} g_{x}(x, y) \sin \left[\frac{k_{y}}{2}\left(y_{12}-y_{11}\right)\right] \cos \left[\frac{k_{y}}{2}\left(y_{12}+y_{11}\right)\right] \cos \left(k_{x} x\right) d x
$$

where $g_{x}(x, y)=(1 / \epsilon(x, y))(d \epsilon(x, y) / d x), \epsilon(x, y)=\epsilon_{0}(1+g(x, y)), k_{x}=(n \pi x) / a$, and $k_{y}=(m \pi y) / b$. Similarly, we can calculate the value of $g_{y}(n, m)$, where $g_{y}(x, y)=(1 / \epsilon(x, y))(d \epsilon(x, y) / d y)$.

For the cross section as shown in Figures 1(a) and 3(b), the center of the rectangle is located at $(0.5 \mathrm{a}, 0.5 \mathrm{~b}), y_{12}=\mathrm{b} / 2+\mathrm{c} / 2$ and $y_{11}=\mathrm{b} / 2-\mathrm{c} / 2$. Thus, for this case, $y_{12}-y_{11}=\mathrm{c}$ and $y_{12}+y_{11}=\mathrm{b}$.

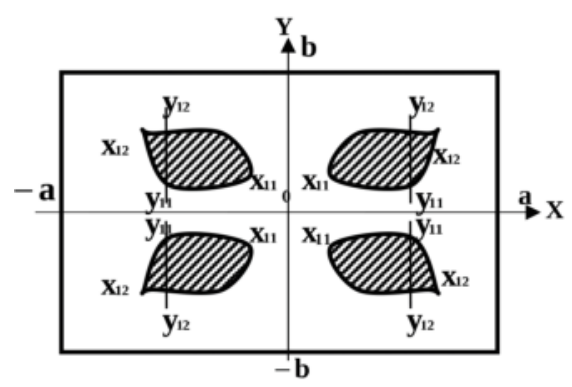

(a)

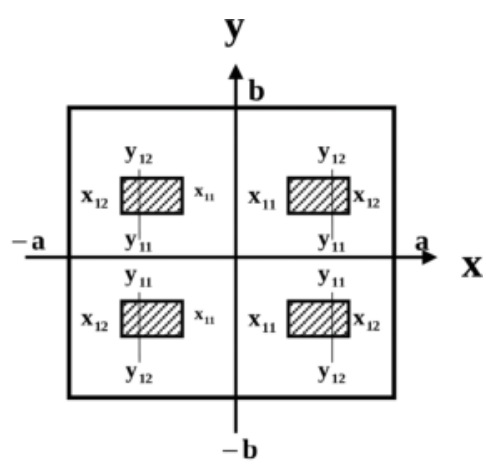

(b)

Figure 3. (a). The arbitrary profile in the cross section of the straight waveguide. (b). The rectangular profile in the cross section of the straight waveguide, according to Figure 1(a). 


\subsection{The technique based on $\omega_{\varepsilon}$ function for the circular profile in the cross section of the straight rectangular waveguide}

The equation of the circle is given by $(x-a / 2)^{2}+(y-b / 2)^{2}=r^{2}$. The center of the circle is located at $(0.5 \mathrm{a}, 0.5 \mathrm{~b})$, as shown in Figure 1(b). We obtain two possibilities without this $y_{11}(x)=b / 2-\sqrt{r^{2}-(x-a / 2)^{2}}$ and $y_{12}(x)=b / 2+\sqrt{r^{2}-(x-a / 2)^{2}}$, where $y_{12}-y_{11}=$ $2 \sqrt{r^{2}-(x-a / 2)^{2}}$ and $y_{12}+y_{11}=b$.

The dielectric profile for the circle is given where the center is located at $(0.5 \mathrm{a}, 0.5 \mathrm{~b})$ (Figure 1(b)) by

$$
g(x, y)= \begin{cases}g_{0} & 0 \leq r<r_{1}-\varepsilon_{1} / 2 \\ g_{0} \exp \left[1-q_{\varepsilon}(r)\right] & r_{1}-\varepsilon_{1} / 2 \leq r<r_{1}+\varepsilon_{1} / 2^{\prime}\end{cases}
$$

where

$$
q_{\varepsilon}(r)=\frac{\varepsilon_{1}^{2}}{\varepsilon_{1}^{2}-\left[r-\left(r_{1}-\varepsilon_{1} / 2\right)\right]^{2}}
$$

else $g(x, y)=0$. The radius of the circle is given by $r=\sqrt{(x-a / 2)^{2}+(y-b / 2)^{2}}$.

The derivatives of the dielectric profile for the circle are given where the center is located at $(0.5 \mathrm{a}$, 0.5 b), as shown in Figure 1(b), where $r_{1}-\varepsilon_{1} / 2 \leq r<r_{1}+\varepsilon_{1} / 2$ by

$$
\begin{aligned}
& g_{x}=\frac{-2 g_{0} \cos \theta \exp \left[1-q_{\varepsilon}(r)\right]\left[r-\left(r_{1}-\varepsilon_{1} / 2\right)\right] \varepsilon_{1}^{2}}{\left\{1+g_{0} \exp \left[1-q_{\varepsilon}(r)\right]\right\}\left[\varepsilon_{1}^{2}-\left[r-\left(r_{1}-\varepsilon_{1} / 2\right)\right]^{2}\right]^{2}}, \\
& g_{y}=\frac{-2 g_{0} \sin \theta \exp \left[1-q_{\varepsilon}(r)\right]\left[r-\left(r_{1}-\varepsilon_{1} / 2\right)\right] \varepsilon_{1}^{2}}{\left\{1+g_{0} \exp \left[1-q_{\varepsilon}(r)\right]\right\}\left[\varepsilon_{1}^{2}-\left[r-\left(r_{1}-\varepsilon_{1} / 2\right)\right]^{2}\right]^{2}},
\end{aligned}
$$

else $g_{x}=0$, and $g_{y}=0$.

The elements of the matrices for the circular profile are given where the center is located at $(0.5 \mathrm{a}$, 0.5 b) (Figure 1(b)) by

$$
\begin{gathered}
g(n, m)=\frac{g_{0}}{a b}\left\{\int_{0}^{2 \pi} \int_{0}^{r_{1}-\varepsilon_{1} / 2} \cos \left[\frac{n \pi}{a}\left(r \cos \theta+\frac{a}{2}\right)\right] \cos \left[\frac{m \pi}{b}\left(r \sin \theta+\frac{b}{2}\right)\right]\right. \\
\left.+\int_{0}^{2 \pi} \int_{r_{1}-\varepsilon_{1} / 2}^{r_{1}+\varepsilon_{1} / 2} \cos \left[\frac{n \pi}{a}\left(r \cos \theta+\frac{a}{2}\right)\right] \cos \left[\frac{m \pi}{b}\left(r \sin \theta+\frac{b}{2}\right)\right] \exp \left[1-q_{\varepsilon}(r)\right]\right\} r d r d \theta, \\
g_{x}(n, m)=-\frac{2 g_{0}}{a b}\left\{\int_{0}^{2 \pi} \int_{r_{1}-\varepsilon_{1} / 2}^{r_{1}+\varepsilon_{1} / 2} \frac{\varepsilon_{1}^{2}\left[r-\left(r_{1}-\varepsilon_{1} / 2\right)\right] \exp \left[1-q_{\varepsilon}(r)\right] \cos \theta}{\left.\left[r-\left(r_{1}-\varepsilon_{1} / 2\right)\right]^{2}\right]}\left[1+g_{0} \exp \left[1-q_{\varepsilon}(r)\right]\right]\right. \\
\left.\cos \left[\frac{n \pi}{a}\left(r \cos \theta+\frac{a}{2}\right)\right] \cos \left[\frac{m \pi}{b}\left(r \sin \theta+\frac{b}{2}\right)\right]\right\} r d r d \theta
\end{gathered}
$$




$$
\begin{aligned}
g_{y}(n, m)=- & \frac{2 g_{0}}{a b}\left\{\int_{0}^{2 \pi} \int_{r_{1}-\varepsilon_{1} / 2}^{r_{1}+\varepsilon_{1} / 2} \frac{\varepsilon_{1}^{2}\left[r-\left(r_{1}-\varepsilon_{1} / 2\right)\right] \exp \left[1-q_{\varepsilon}(r)\right] \sin \theta}{\left[\varepsilon_{1}{ }^{2}-\left[r-\left(r_{1}-\varepsilon_{1} / 2\right)\right]^{2}\right]^{2}\left[1+g_{0} \exp \left[1-q_{\varepsilon}(r)\right]\right]}\right. \\
& \left.\cos \left[\frac{n \pi}{a}\left(r \cos \theta+\frac{a}{2}\right)\right] \cos \left[\frac{m \pi}{b}\left(r \sin \theta+\frac{b}{2}\right)\right]\right\} r d r d \theta,
\end{aligned}
$$

where $r=\sqrt{(x-a / 2)^{2}+(y-b / 2)^{2}}$.

The proposed techniques in this subsection and the subsection 3.1 relate to the method for the propagation along the straight rectangular metallic waveguide [32]. The techniques and the particular applications to solve the rectangular and circular profiles in the cross section of the straight rectangular waveguide are important in order to improve the mode model [32]. The method is based on the Laplace and Fourier transform and their inverse transforms. Laplace transform is necessary to obtain the comfortable and simple input-output connections of the fields. The output transverse field profiles are computed by the inverse Laplace and Fourier transforms.

The matrix $\mathrm{G}$ is given by the form.

$$
\mathbf{G}=\left[\begin{array}{ccccccc}
g_{00} & g_{-10} & g_{-20} & \cdots & g_{-n m} & \cdots & g_{-N M} \\
g_{10} & g_{00} & g_{-10} & \cdots & g_{-(n-1) m} & \cdots & g_{-(N-1) M} \\
g_{20} & g_{10} & \ddots & \ddots & \ddots & & \\
\vdots & g_{20} & \ddots & \ddots & \ddots & & \\
g_{n m} & \ddots & \ddots & \ddots & g_{00} & \vdots & \\
\vdots & & & & & & \\
g_{N M} & \cdots & \cdots & \cdots & \cdots & \cdots & g_{00}
\end{array}\right] .
$$

Similarly, the $\mathbf{G}_{x}$ and $\mathbf{G}_{y}$ matrices are obtained by the derivatives of the dielectric profile. These matrices relate to the method that is based on the Laplace and Fourier transforms and their inverse [32].

Several examples will demonstrate in the next section in order to explore the effects of the rectangular and circular materials in the cross section (Figure 1(a) and 1(b)) along the straight waveguide on the output field. All the graphical results will be demonstrated as a response to a half-sine $\left(T E_{10}\right)$ input-wave profile and the rectangular and circular materials in the cross section of the straight rectangular waveguide.

\subsection{The technique based on $\omega_{\varepsilon}$ function for the circular profile in the cross section of the straight circular waveguide}

The cross section of hollow waveguide (Figure 1(c)) is made of a tube of various types of three dielectric layers and a metallic layer. The internal and external diameters are denoted as $2 b$, 
$2 b_{1}, 2 b_{2}, 2 \mathrm{a}$, and $2\left(\mathrm{a}+\delta_{m}\right)$, respectively, where $\delta_{m}$ is the thickness of the metallic layer. In addition, we denote the thickness of the dielectric layers as $d_{1}, d_{2}$, and $d_{3}$, respectively, where $d_{1}=b_{1}-\mathrm{b}, d_{2}=b_{2}-b_{1}$, and $d_{3}=\mathrm{a}-b_{2}$. The refractive index in the particular case with the three dielectric layers and the metallic layer in the cross section of the straight hollow waveguide (Figure 1(c)) is calculated as follows:

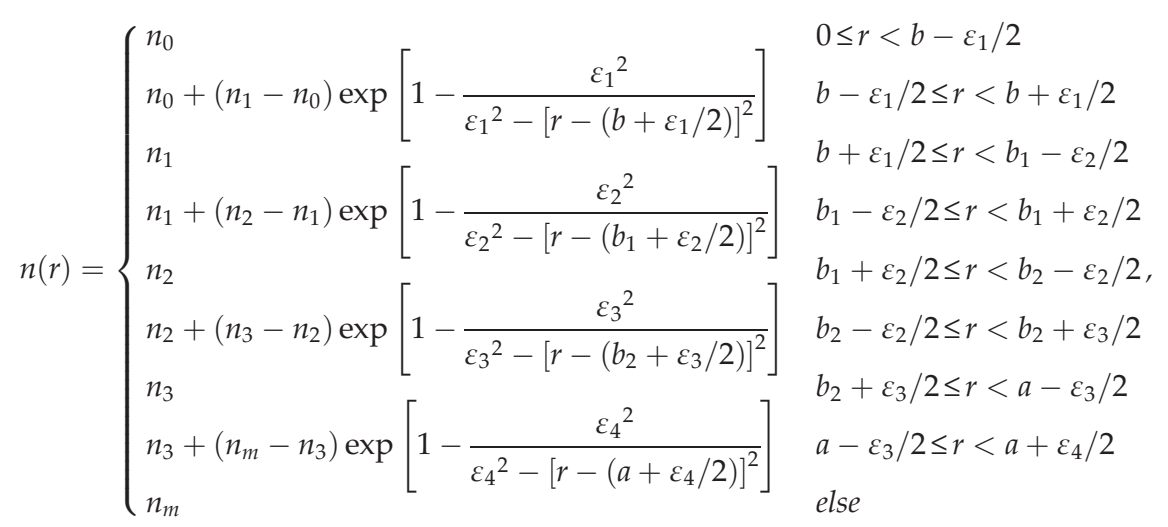

where the parameters $\varepsilon_{1}, \varepsilon_{2}, \varepsilon_{3}$, and $\varepsilon_{4}$ are very small [e.g., $\left.\varepsilon_{1}=\varepsilon_{2}=\varepsilon_{3}=[a-b] / 50, \varepsilon_{4}=\delta_{m} / 50\right]$. The refractive indices of the air, dielectric and metallic layers are denoted as $n_{0}, n_{1}, n_{2}, n_{3}$, and $n_{m}$, respectively. In this study, we suppose that $n_{3}>n_{2}>n_{1}$.

The transverse derivative of the dielectric profile is calculated as follows:

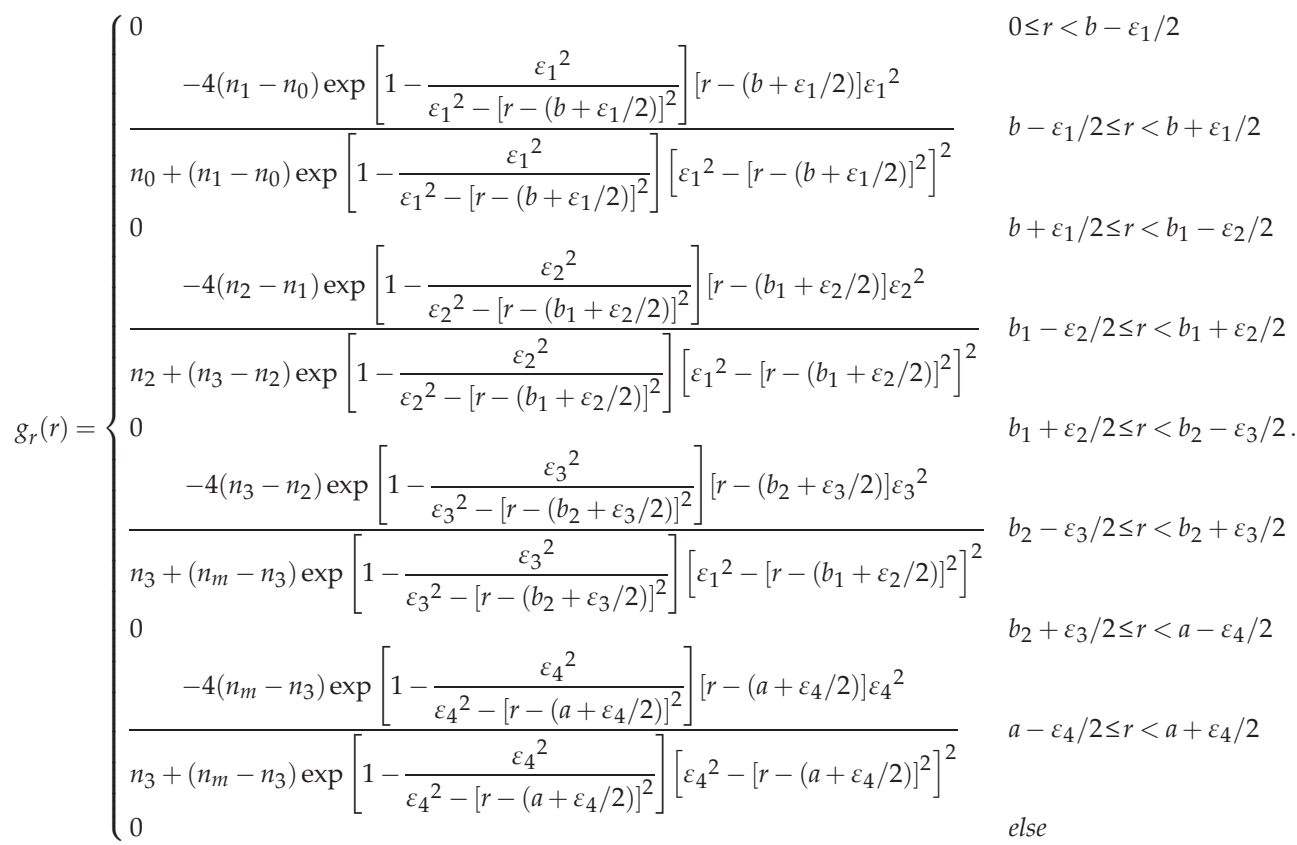


The proposed technique in this subsection relates to the theoretical model that based on Maxwell's equations, Fourier-Bessel series, Laplace transform, and the inverse Laplace transform [33]. In this theoretical model, the longitudinal components of the fields are developed into the Fourier-Bessel series. The transverse components of the fields are expressed as functions of the longitudinal components in the Laplace plane and are obtained by using the inverse Laplace transform by the residue method. The separation of the variables is obtained by using the orthogonal relations.

The output transverse components of the fields of the straight hollow waveguide are finally expressed in a form of transfer matrix function, and the derivation has been already explained in detail in Ref. [33]. The contribution of the proposed technique to calculate the refractive indices $(n(r))$ and the transverse derivative of the dielectric profile $\left(g_{r}\right)$ for three dielectric layers is important to improve the method that is based on the mode model [33]. This improved method is important to reduce the transmission losses of the dielectric coated metal waveguides.

\section{Numerical results}

Several examples for all geometry of the rectangular and circular waveguides and the dielectric profile are demonstrated in this section for three cases, as shown in Figure 1(a)-(c).

\subsection{Numerical results for the rectangular dielectric material in the cross section of the straight rectangular waveguide}

The analytical method for the dielectric slab [35] is shown in Figure 4(a). The slab profile in the cross section is based on transcendental equation, as follows:

$$
\begin{gathered}
E_{y 1}=j \frac{k_{z}}{\varepsilon_{0}} \sin (v x) \quad 0<x<t \\
E_{y 2}=j \frac{k_{z}}{\varepsilon_{0}} \frac{\sin (v t)}{\cos (\mu(t-a / 2))} \cos [\mu(x-a / 2)] \quad t<x<t+d \\
E_{y 3}=j \frac{k_{z}}{\varepsilon_{0}} \sin [v(a-x)] \quad t+d<x<a,
\end{gathered}
$$

where $v \equiv \sqrt{k_{o}^{2}-k_{z}^{2}}$ and $\mu \equiv \sqrt{\varepsilon_{r} k_{o}^{2}-k_{z}^{2}}$ result from the transcendental equation.

$$
\left(\frac{a-d}{d}\right) \frac{d \mu}{2} \tan \left(\frac{d \mu}{2}\right)-(t v) \cot (t v)=0 .
$$

The criterion for the convergence of the solution is given by 


$$
C(N) \equiv \log \left\{\frac{\max \left(\left|E_{y}^{N+2}-E_{y}^{N}\right|\right)}{\left|\max \left(E_{y}^{N+2}\right)-\min \left(E_{y}^{N}\right)\right|}\right\}
$$

for $N \geq 1$, where $(2 N+1)^{2}$ is the number of the modes. By increasing the order $N$, then $E_{y}(N)$ approaches $E_{y}$.

Figure 4(a) shows the geometry of the slab profile for practical case of the slab dielectric material, where $\mathrm{a}=20 \mathrm{~mm}, \mathrm{~b}=10 \mathrm{~mm}, \mathrm{~d}=3.3 \mathrm{~mm}, \mathrm{t}=8.35 \mathrm{~mm}, \lambda=6.9 \mathrm{~cm}$, and $\epsilon_{r}=9$.

Figure 4(b) demonstrates the output result of the comparison between the theoretical model with the analytical solution [35].

Figure 4(c) shows the criterion of the convergence in Eq. (18). Between $N=7$ and $N=9$ the value of the criterion is equal to -2 . According to Figure $4(\mathbf{b})$ and $4(c)$, the comparison has shown good agreement.

All the next graphical results are demonstrated as a response to a half-sine ( $\left.T E_{10}\right)$ input-wave profile.

Figure 5(a)-(f) relates to discontinuous problem according to Figure 1(a). Figure 5(a)-(d) shows the results of the output field as a response to a half-sine $\left(T E_{10}\right)$ input-wave profile. In this case, $\mathrm{c}=\mathrm{d}=3.3 \mathrm{~mm}$ and the center of the rectangle is located at the point $(0.5 \mathrm{a}, 0.5 \mathrm{~b})$, as shown in Figure 1 (a) for $\epsilon_{r}=3,5,7$, and 10, respectively. The other parameters are $\mathrm{a}=\mathrm{b}=2 \mathrm{~cm}, \mathrm{z}=15 \mathrm{~cm}, k_{0}=1671 / \mathrm{m}$, $\lambda=3.75 \mathrm{~cm}$, and $\beta=581 / \mathrm{m}$. The output fields are strongly affected by the input wave profile $\left(T E_{10}\right.$ mode), the rectangular profile, and the location of the center of the rectangle $(0.5 \mathrm{a}, 0.5 \mathrm{~b})$.

Figure 5(e) shows the output amplitude and the Gaussian shape of the central peak in the same cross section of Figure 5(a)-(d), where $a=2 \mathrm{~cm}, b=2 \mathrm{~cm}, y=b / 2=10 \mathrm{~mm}, c=3.3 \mathrm{~mm}$, $\mathrm{d}=3.3 \mathrm{~mm}, \mathrm{z}=15 \mathrm{~cm}, k_{0}=1671 / \mathrm{m}$, and for $\epsilon_{r}=3,5,7$, and 10, respectively.

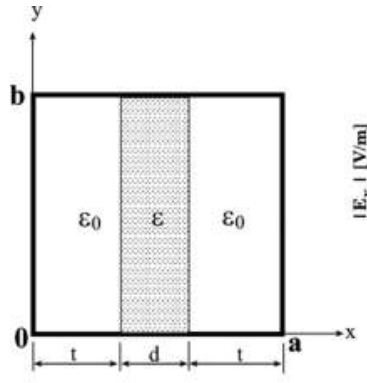

(a)

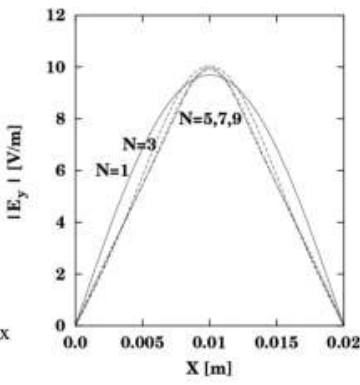

(b)

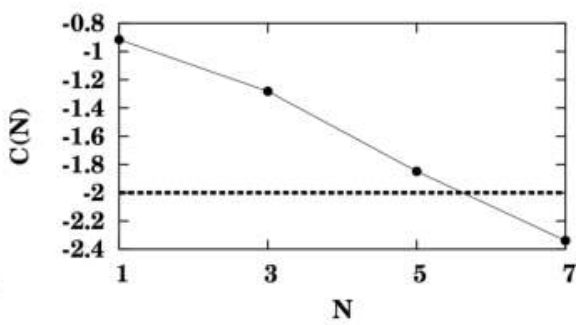

(c)

Figure 4. (a). The cross section of the straight rectangular waveguide. (b). The results between our model and the analytical method. (c). The criterion of the convergence according to Eq. (18). 


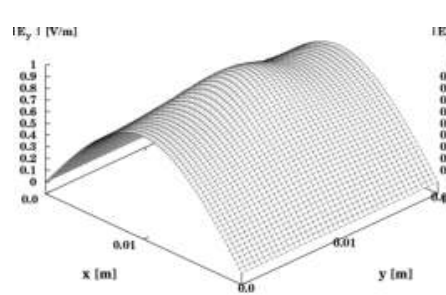

(a)

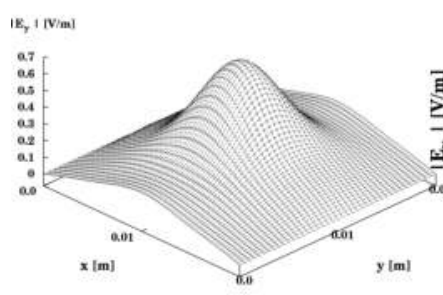

(d)

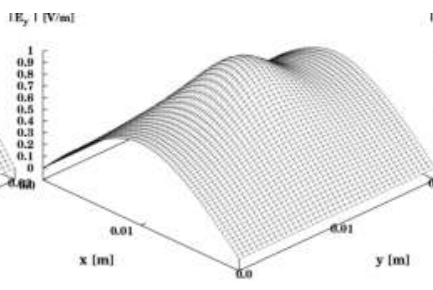

(b)

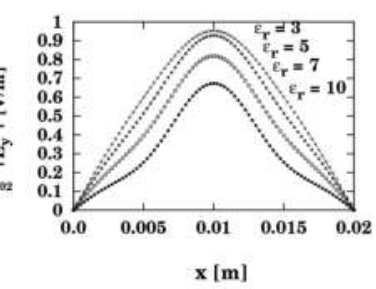

(e)

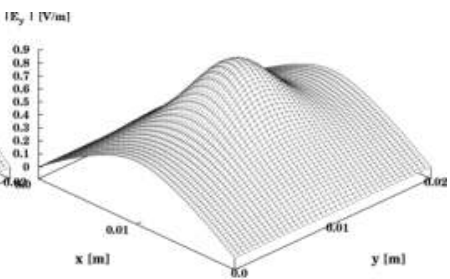

(c)

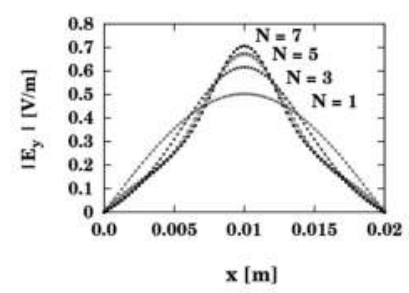

(f)

Figure 5. The output field as a response to a half-sine $\left(T E_{10}\right)$ input-wave profile where $\mathrm{c}=\mathrm{d}=3.3 \mathrm{~mm}$ and the center of the rectangle is located at the point $(0.5 \mathrm{a}, 0.5 \mathrm{~b})$ according to Figure $1(\mathrm{a})$, where: (a). $\epsilon_{r}=3 ;$ (b). $\epsilon_{r}=5 ;$ (c). $\epsilon_{r}=7 ;(\mathrm{d}) . \epsilon_{r}=10$. The other parameters are $\mathrm{a}=\mathrm{b}=2 \mathrm{~cm}, \mathrm{z}=15 \mathrm{~cm}, k_{0}=1671 / \mathrm{m}, \lambda=3.75 \mathrm{~cm}$, and $\beta=581 / \mathrm{m}$. (e). The output field for $\epsilon_{r}=3,5,7$, and 10 , respectively, where $\mathrm{a}=\mathrm{b}=2 \mathrm{~cm}, \mathrm{y}=\mathrm{b} / 2=10 \mathrm{~mm}$. (f). The output profiles for $\mathrm{N}=1,3,5$, and 7 , where $\epsilon_{r}=10$.

By increasing only the value of $\epsilon_{r}$ of the rectangular dielectric profile, the $T E_{10}$ wave profile decreased, the Gaussian shape of the output field increased, and the relative amplitude decreased. In addition, by increasing only the value of $\epsilon_{r}$, the width of the Gaussian shape decreased.

The output profiles and the amplitudes for $\mathrm{N}=1,3,5$, and 7 are shown in Figure 5(f), for $\epsilon_{r}=$ 10. By increasing only the parameter of the order $\mathrm{N}$, the output field approaches to the final output field.

According to the results, we see that the output fields are strongly affected by the input wave profile ( $T E_{10}$ mode), the rectangular profile, and the location of the center of the rectangle $(0.5 \mathrm{a}, 0.5 \mathrm{~b})$.

\subsection{Numerical results for the circular dielectric material in the cross section of the straight rectangular waveguide}

Figure 6(a)-(f) relates to discontinuous problem according to Figure 1(b). Figure 6(a)-(d) demonstrates the results of the output field as a response to $T E_{10}$ input-wave profile, for $\epsilon_{r}=$ $3,5,7$, and 10, respectively. The radius of the circle in this case is equal to $2.5 \mathrm{~mm}$ and the center of the circle is located at the point $(0.5 \mathrm{a}, 0.5 \mathrm{~b})$, as shown in Figure 1(b).

The other parameters are $\mathrm{a}=\mathrm{b}=2 \mathrm{~cm}, \mathrm{z}=15 \mathrm{~cm}, k_{0}=1671 / \mathrm{m}, \lambda=3.75 \mathrm{~cm}$, and $\beta=581 / \mathrm{m}$.

Figure 6(e) shows the output amplitude and the Gaussian shape of the central peak in the same cross section of Figure 6(a)-(d), where $\epsilon_{r}=3,5,7$, and 10, respectively. 


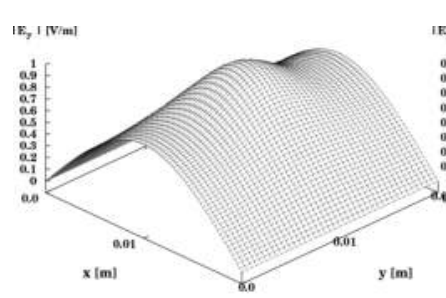

(a)

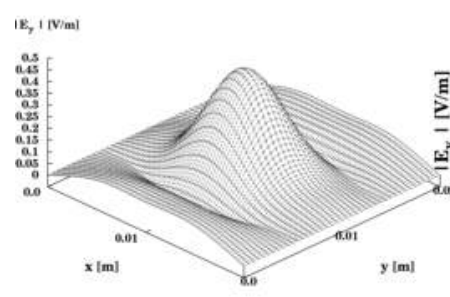

(d)

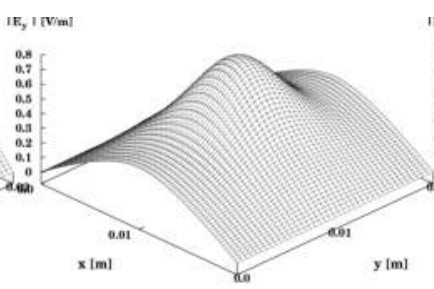

(b)

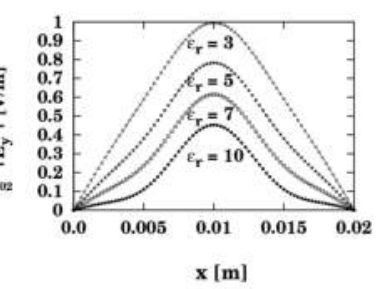

(e)

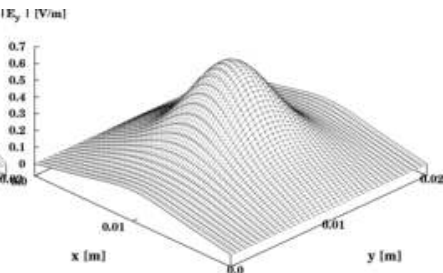

(c)

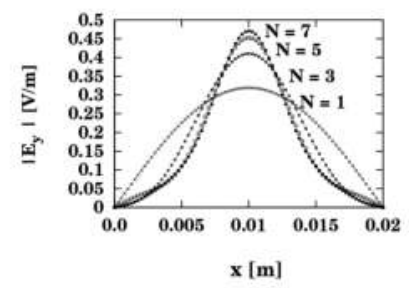

(f)

Figure 6. The output field as a response to a half-sine $\left(T E_{10}\right)$ input-wave profile where the radius of all circle is equal to $2.5 \mathrm{~mm}$ and the center of the circle is located at the point $(0.5 \mathrm{a}, 0.5 \mathrm{~b})$ according to Figure 1(b), where: (a). $\epsilon_{r}=3 ;$ (b). $\epsilon_{r}=5 ;$ (c). $\epsilon_{r}=7 ;(\mathrm{d})$. $\epsilon_{r}=10$. The other parameters are $\mathrm{a}=\mathrm{b}=2 \mathrm{~cm}, \mathrm{z}=15 \mathrm{~cm}, k_{0}=1671 / \mathrm{m}, \lambda=3.75 \mathrm{~cm}$, and $\beta=581 / \mathrm{m}$. (e). The output field for $\epsilon_{r}=3$, 5,7 , and 10 , respectively, where $\mathrm{a}=\mathrm{b}=20 \mathrm{~mm}, \mathrm{y}=\mathrm{b} / 2=10 \mathrm{~mm}$. The radius of all circles is equal to $2.5 \mathrm{~mm}$. (f). The output profiles for $\mathrm{N}=1,3,5$, and 7 , where $\epsilon_{r}=10$.

The output profiles are shown in Figure $6(\mathbf{f})$ for $\mathrm{N}=1,3,5$, and 7 , where $\epsilon_{r}=10$. The other parameters are $\mathrm{a}=\mathrm{b}=2 \mathrm{~cm}, \mathrm{z}=15 \mathrm{~cm}, k_{0}=1671 / \mathrm{m}, \lambda=3.75 \mathrm{~cm}$, and $\beta=581 / \mathrm{m}$.

By increasing only the parameter $\epsilon_{r}$ of the circular dielectric profile from 3 to 10, the Gaussian shape of the output transverse profile of the field increased, the $T E_{10}$ wave profile decreased, and the relative amplitude of the output field decreased.

The output fields of Figure 6(a)-(f) are strongly affected by the input wave profile ( $T E_{10}$ mode), the circular profile, and the location of the center of the circle $(0.5 \mathrm{a}, 0.5 \mathrm{~b})$.

It is interesting to see a similar behavior of the output results in the cases of rectangular profiles (Figure 5(a)-(f)) that relate to Figure 1(a) and in the cases of circular profiles (Figure 6(a)-(f)) that relate to Figure 1(b), for every value of $\epsilon_{r}$, respectively. According to these output results, we see the similar behavior for every value of $\epsilon_{r}$, but the amplitudes of the output fields are different.

\subsection{Numerical results for the three dielectric layers and a metallic layer in the cross section of the straight hollow waveguide}

The comparison between our theoretical result of the output power density with the laboratory result in the case of the straight hollow waveguide with one dielectric layer and a metallic layer 
is demonstrated in Figure $\mathbf{7}(\mathbf{a})$ and $\mathbf{7}(\mathbf{b})$. In this example, the diameter (2a) of the waveguide is $2 \mathrm{~mm}$, the thickness of the dielectric layer $\left[d_{(A g I)}\right]$ is $0.75 \mu \mathrm{m}$, and the minimum spot-size $\left(w_{0}\right)$ is $0.3 \mathrm{~mm}$. The length of the straight waveguide is $1 \mathrm{~m}$. The refractive indices of the air, the dielectric layer (AgI) and the metallic layer $(\mathrm{Ag})$ are $n_{(0)}=1, n_{(A g I)}=2.2$, and $n_{(A g)}=$ $13.5-j 75.3$, respectively. The value of the refractive index of the material at a wavelength of $\lambda=10.6 \mu \mathrm{m}$ is taken from the table compiled by Miyagi et al. [36].

The results of the output power density $\left(\left|S_{a v}\right|\right)$ (e.g., Figure 7(a)) show the behavior of the solutions for the TEM 00 mode in excitation. The comparison between our theoretical result (Figure 7(a)) and the published experimental data [37], as shown also in Figure 7(b) shows good agreement of a Gaussian shape as expected, except for the secondary small propagation mode. The experimental result is taken into account the roughness of the internal wall of the waveguide, but our theoretical model is not taken the roughness.

Miyagi and Kawakami showed that transmission losses of the dielectric-coated metal waveguides are drastically reduced when a multiple dielectric layer is formed instead of a single dielectric layer [31]. The simplest and most efficient multilayer structure is three dielectric layers that deposited on a metal layer.

Thus, in this subsection, we present the output results that relate to the proposed technique and the particular application for the cross section of the straight hollow waveguide with three dielectric layers (and not only with one dielectric layer) and a metallic layer. In this case, we can improve the method [33], in the case of the three dielectric layers and a metallic layer in the cross section of the straight hollow waveguide.

Figure 8(a)-(c) relates to discontinuous problem according to Figure 1(c). The output power density for the straight hollow waveguide with three dielectric layers is shown in Figure 8(a)(c). Figure $8(\mathbf{a})$ is shown for $\mathrm{a}=0.8 \mathrm{~mm}$ and $w_{0}=0.1 \mathrm{~mm}$. Figure $\mathbf{8}(\mathbf{b})$ is shown for a $=0.8 \mathrm{~mm}$ and $w_{0}=0.3 \mathrm{~mm}$. Figure $8(\mathbf{c})$ is shown for $\mathrm{a}=0.6 \mathrm{~mm}$ and $w_{0}=0.3 \mathrm{~mm}$. The other parameters are $\mathrm{b}=0.5 \mathrm{~mm}, \lambda=10.6 \mu \mathrm{m}, n_{(0)}=1, n_{1}=2.22-\mathrm{j} 10^{-6}, n_{2}=4-\mathrm{j} 10^{-6}, n_{3}=6-\mathrm{j} 10^{-6}, n_{m}=n_{(\mathrm{Ag})}=$ $13.5-\mathrm{j} 75.3$, and the length of the straight waveguide is $1 \mathrm{~m}$.

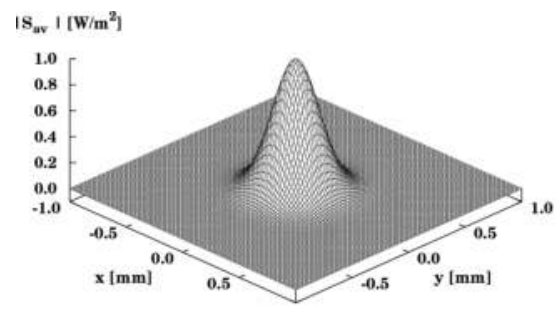

(a)

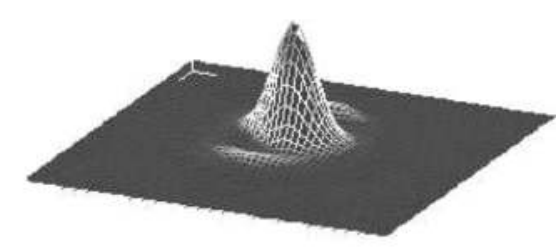

(b)

Figure 7. The comparison between our theoretical results of the output power density with the laboratory results in the case of the straight hollow waveguide with one dielectric layer. The parameters are a $=1 \mathrm{~mm}, w_{0}=0.3 \mathrm{~mm}, d_{(A g I)}=0.75$ $\mu m, \lambda=10.6 \mu m, n_{(0)}=1, n_{(A g I)}=2.2, n_{(A g)}=13.5-\mathrm{j} 75$, and the length of the straight waveguide is $1 \mathrm{~m}$. (a). theoretical result. (b). laboratory result. 


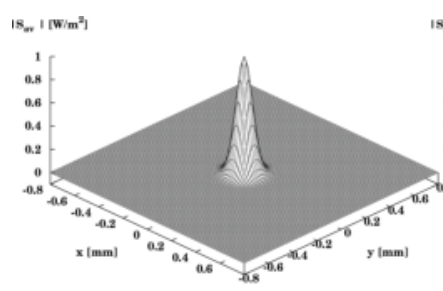

(a)

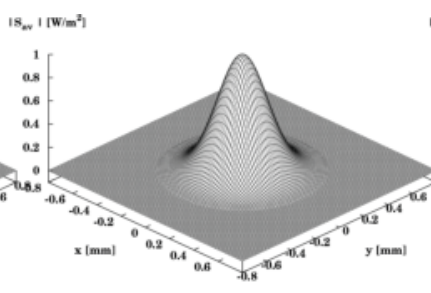

(b)

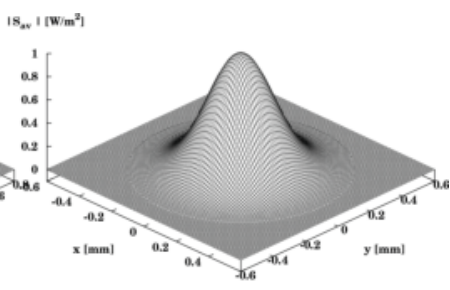

(c)

Figure 8. The output power density for the straight hollow waveguide with three dielectric layers, where (a). $\mathrm{a}=0.8 \mathrm{~mm}$ and $w_{0}=0.1 \mathrm{~mm}$. (b). $\mathrm{a}=0.8 \mathrm{~mm}$ and $w_{0}=0.3 \mathrm{~mm}$. (c). $\mathrm{a}=0.6 \mathrm{~mm}$ and $w_{0}=0.3 \mathrm{~mm}$. The other parameters are: $\mathrm{b}=0.5 \mathrm{~mm}$, $\lambda=10.6 \mu m, n_{(0)}=1, n_{1}=2.22-\mathrm{j} 10^{-6}, n_{2}=4-\mathrm{j} 10^{-6}, n_{3}=6-\mathrm{j} 10^{-6}, n_{m}=n_{(\mathrm{Ag})}=13.5-\mathrm{j} 75.3$, and the length of the straight waveguide is $1 \mathrm{~m}$.

By changing only the spot size from $w_{0}=0.1$ to $0.3 \mathrm{~mm}$, with the same other parameters, the output power density is changed, as shown in Figure 8(a) and 8(b). The results of the output power density $\left(\left|S_{a v}\right|\right)$ show the behavior of the solutions for the $T E M_{00}$ mode in excitation. By changing only the spot size from $w_{0}=0.3$ to $0.1 \mathrm{~mm}$, the width of the output Gaussian becomes more narrow. The output field results are strongly affected by the spot size and the structure of the three layers and the metallic layer in the cross section of the straight hollow waveguide.

This mode model can be a useful tool to predict the relevant parameters in the case of the hollow waveguide with three dielectric layers and a metallic layer (Ag) for practical applications (output fields, output power density and output power transmission), before carrying out experiments in the laboratory.

\section{Conclusions}

The main objective of this chapter was to develop the techniques that enable us to solve problems with inhomogeneous dielectric materials in the cross section of the straight rectangular and circular waveguides. The proposed techniques are very effective in relation to the conventional methods because they allow the development of expressions in the cross section only according to the specific discontinuous problem. In this way, the mode model methods become an improved methods to solve discontinuous problems in the cross section (and not only for continuous problems).

Three examples of inhomogeneous dielectric profiles in the cross section of the straight waveguides were shown in Figure 1(a)-(c). Figure 1(a) and 1(b) show the rectangular and circular profiles in the cross section of the straight rectangular waveguide, respectively. Figure 1(c) shows three dielectric layers and a metallic layer in the cross section of the straight hollow waveguide. The second objective is to understand the influence of the inhomogeneous dielectric materials on the output fields.

The proposed techniques are important to improve the methods that are based on Laplace and Fourier transforms and their inverse transforms also for the discontinuous rectangular and 
circular profiles in the cross section. The technique based on $\omega_{\varepsilon}$ function was explained in detail in this chapter.

The result of the comparison between the theoretical models with the analytical solution [35] is shown in Figure 4(b) and the convergence is shown in Figure 4(c). The comparison has shown good agreement.

The results for the rectangular profile in the cross section of the straight rectangular waveguide (Figure 1(a)) were shown in Figure 5(a)-(f). Figure 5(a)-(d) shows the results of the output field as a response to a half-sine $\left(T E_{10}\right)$ input-wave profile. The output fields are strongly affected by the input wave profile ( $T E_{10}$ mode), the rectangular profile, and the location of the center of the rectangle $(0.5 \mathrm{a}, 0.5 \mathrm{~b})$.

By increasing only the value of $\epsilon_{r}$ of the rectangular dielectric material, the Gaussian shape of the output field increased, the $T E_{10}$ wave profile decreased, the relative amplitude decreased, and the width of the Gaussian shape decreased. The output field approaches to the final output field, by increasing only the parameter of the order N, as shown in Figure 5(f).

The results for the circular profile in the cross section of the straight rectangular waveguide (Figure 1(b)) were shown in Figure 6(a)-(f). Figure 6(a)-(d) shows the results of the output field as a response to a half-sine $\left(T E_{10}\right)$ input-wave profile.

By increasing only the value of the parameter $\epsilon_{r}$ of the circular dielectric material (Figure 1(b)) in the rectangular cross section from 3 to 10, the Gaussian shape of the output transverse profile of the field increased, the $T E_{10}$ wave profile decreased, and the relative amplitude of the output field decreased.

The output fields of Figure 5(a)-(f) and Figure 6(a)-(f) are strongly affected by the input wave profile ( $T E_{10}$ mode), the rectangular profile or circular profile, and the location of the center of the rectangle or the circle $(0.5 \mathrm{a}, 0.5 \mathrm{~b})$.

It is interesting to see a similar behavior of the output results in the cases of rectangular profiles (Figure 5(a)-(f)) that relate to Figure 1(a) and in the cases of circular profiles (Figure 6(a)-(f)) that relate to Figure 1(b), for every value of $\epsilon_{r}$, respectively. According to these output results, we see the similar behavior for every value of $\epsilon_{r}$, but the amplitudes of the output fields are different.

The comparison between our theoretical result (Figure 7(a)) and the published experimental data [37], as shown also in Figure 7(b) shows good agreement of a Gaussian shape as expected, except for the secondary small propagation mode. The experimental result is taken into account the roughness of the internal wall of the waveguide, but our theoretical model is not taken the roughness.

The output power density for the straight hollow waveguide with three dielectric layers (Figure 1(c)) is shown in Figure 8(a)-(c). The output field results are strongly affected by the spot size and the structure of the three layers and the metallic layer in the cross section of the straight hollow waveguide.

These models are useful to predict the structure of the output fields for rectangular and circular profiles in straight waveguides in the cases of microwave and millimeter-wave regimes and in 
the cases of infrared regime. These models can be a useful tool to predict the relevant parameters for practical applications, before carrying out experiments in the laboratory.

\section{Author details}

Zion Menachem

Address all correspondence to: zionm@post.tau.ac.il

Department of Electrical Engineering, Sami Shamoon College of Engineering, Beer Sheva, Israel

\section{References}

[1] Chiang KS. Review of numerical and approximate methods for the modal analysis of general optical dielectric waveguides. Optical and Quantum Electronics. 1993;26:S113S134

[2] Zhao S. Full-vectorial matched interface and boundary (MIB) method for the modal analysis of dielectric waveguides. Journal of Lightwave Technology. 2008;26:2251-2259

[3] Harrington JA, Matsuura Y. Review of hollow waveguide technology. SPIE. 1995;2396: $4-14$

[4] Harrington JA, Harris DM, Katzir A, editors. Biomedical Optoelectronic Instrumentation. 1995;2396:4-14

[5] Harrington JA. A review of IR transmitting, hollow waveguides. Fiber and Integrated Optics. 2000;19:211-228

[6] Marhic ME. Mode-coupling analysis of bending losses in IR metallic waveguides. Applied Optics. 1981;20:3436-3441

[7] Croitoru N, Goldenberg E, Mendlovic D, Ruschin S, Shamir N. Infrared chalcogenide tube waveguides. SPIE. 1986;618:140-145

[8] Jiao CQ. Selective suppression of electromagnetic modes in a rectangular waveguide by using distributed wall losses. Progress in Electromagnetics Research Letters. 2011;22:119128

[9] Mineo M, Di Carlo A, Paoloni C. Analytical design method for corrugated rectangular waveguide SWS THZ vacuum tubes. Journal of Electromagnetic Waves and Applications. 2010;24:2479-2494

[10] Smartt CJ, Benson TM, Kendall PC. Exact transcendental equation for scalar modes of rectangular dielectric waveguides. Optical and Quantum Electronics. 1994;26:641-644 
[11] Chen TT. Wave propagation in an inhomogeneous transversely magnetized rectangular waveguide. Applied Scientific Research. 1960;8:141-148

[12] Yeap KH, Tham CY, Yassin G, Yeong KC. Attenuation in rectangular waveguides with finite conductivity walls. Radioengineering. 2011;20:472-478

[13] Sharma J. Full-wave analysis of dielectric rectangular waveguides. Progress In Electromagnetics Research. 2010;13:121-131

[14] Sumathy M, Vinoy KJ, Datta SK. Analysis of rectangular folded-waveguide millimeterwave slow-wave structures using conformal transformations. Journal of Infrared, Millimeter, and Terahertz Waves. 2009;30:294-301

[15] Rothwell EJ, Temme A, Crowgey B. Pulse reflection from a dielectric discontinuity in a rectangular waveguide. Progress In Electromagnetics Research. 2009;97:11-25

[16] Liu S, Li LW, Leong MS, Yeo TS. Rectangular conducting waveguide filled with uniaxial anisotropic media: a modal analysis and dyadic Green's function. Progress In Electromagnetics Research. 2000;25:111-129

[17] Han SH, Wang XL, Fan Y. Improved generalized admittance matrix technique and its applications to rigorous analysis of millimeter-wave devices in rectangular waveguide. International Journal of Infrared and Millimeter Waves. 2006;27:1391-1402

[18] Lu W, Lu YY. Waveguide mode solver based on Neumann-to-Dirichlet operators and boundary integral equations. Journal of Computational Physics. 2012;231:1360-1371

[19] Eyges L, Gianino P. Modes of dielectric waveguides of arbitrary cross sectional shape. Journal of the Optical Society of America. 1979;69:1226-1235

[20] Abbas Z, Pollard RD, Kelsall W. A rectangular dielectric waveguide technique for determination of permittivity of materials at W-band. IEEE Transactions on Microwave Theory and Techniques. 1998;46:2011-2015

[21] Hewlett SJ, Ladouceur F. Fourier decomposition method applied to mapped infinite domains: scalar analysis of dielectric waveguides down to modal cutoff. Journal of Lightwave Technology. 1995;13:375-383

[22] Hernandez-Lopez MA, Quintillan M. Propagation characteristics of modes in some rectangular waveguides using the finite-difference time-domain method. Journal of Electromagnetic Waves and Applications. 2000;14:1707-1722

[23] Vaish A, Parthasarathy H. Analysis of rectangular waveguide using finite element method. Progress In Electromagnetics Research C. 2008;2:117-125

[24] Baganas K. Inhomogeneous dielectric media: Wave propagation and dielectric permittivity reconstruction in the case of a rectangular waveguide. Journal of Electromagnetic Waves and Applications. 2002;16:1371-1392

[25] Novotny L, Hafner C. Light propagation in a cylindrical waveguide with a complex, metallc, dielectric function. Physical Review E. 1994;50:4094-4106 
[26] Yener N. Advancement of algebraic function approximation in eigenvalue problems of lossless metallic waveguides to infinite dimensions, part I: Properties of the operator in infinite dimensions. Journal of Electromagnetic Waves and Applications. 2006;20:16111628

[27] Yener N. Algebraic function approximation in eigenvalue problems of lossless metallic waveguides: Examples. Journal of Electromagnetic Waves and Applications. 2006;20:731745

[28] Khalaj-Amirhosseini M. Analysis of longitudinally inhomogeneous waveguides using Taylor's series expansion. Journal of Electromagnetic Waves and Applications. 2006;20: $1093-1100$

[29] Khalaj-Amirhosseini M. Analysis of longitudinally inhomogeneous waveguides using the Fourier series expansion. Journal of Electromagnetic Waves and Applications. 2006;20: $1299-1310$

[30] Reutskiy SY. The methods of external excitation for analysis of arbitrarily-shaped hollow conducting waveguides. Progress in Electromagnetics Research. 2008;82:203-226

[31] Miyagi M, Kawakami S. Design theory of dielectric-coated circular metallic waveguides for infrared transmission. Journal of Lightwave Technology. 1984;LT-2:116-126

[32] Menachem Z, Jerby E. Transfer matrix function (TMF) for propagation in dielectric waveguides with arbitrary transverse profiles. IEEE Transactions on Microwave Theory and Techniques. 1998;46:975-982

[33] Menachem Z, Tapuchi S. Influence of the spot-size and cross-section on the output fields and power density along the straight hollow waveguide. Progress in Electromagnetics Research. 2013;48:151-173

[34] Vladimirov V. Equations of Mathematical Physics. New York: Marcel Dekker, Inc.; 1971

[35] Collin RE. Foundation for Microwave engineering. New York: McGraw-Hill; 1996

[36] Miyagi M, Harada K, Kawakami S. Wave propagation and attenuation in the general class of circular hollow waveguides with uniform curvature. IEEE Transactions on Microwave Theory and Techniques. 1984;32:513-521

[37] Croitoru N, Inberg A, Oksman M, Ben-David M. Hollow silica, metal and plastic waveguides for hard tissue medical applications. SPIE. 1997;2977:30-35 
